\title{
Evidence for the non-destruction of the Most Massive Molecular Clouds even after they have given Birth to Massive Star Clusters
}

\author{
J. Zaragoza-Cardiel ${ }^{1}$, J. Beckman ${ }^{2,3,4}$, J. Font ${ }^{2,3}$, A. Camps-Fariña ${ }^{2,3}$, \\ B. García-Lorenzo ${ }^{2,3}$ and A. Serrano-Borlaff ${ }^{2,3}$ \\ ${ }^{1}$ Instituto de Astronomía, Universidad Nacional Autónoma de México, Mexico City, Mexico \\ email: javier.zaragoza@astro.unam.mx \\ ${ }^{2}$ Instituto de Astrofísica de Canarias, C/ Vía Láctea s/n, 38205 La Laguna, Tenerife, Spain \\ ${ }^{3}$ Department of Astrophysics, University of La Laguna, E-38200 La Laguna, Tenerife, Spain \\ ${ }^{4}$ CSIC, 28006 Madrid, Spain
}

\begin{abstract}
We have combined observations of the Antennae galaxies from the radio interferometer ALMA and from the optical interferometer GH $\alpha$ FaS (Galaxy $\mathrm{H} \alpha$ Fabry-Perot System). The two sets of observations have comparable angular and spectral resolutions, enabling us to identify 142 giant molecular clouds (GMCs) and 303 Hil regions. We have measured, and compared, their basic physical properties (radius, velocity dispersion, luminosity). We study the mass functions of HII regions and GMCs, where a double population of Hil regions due to a double population of GMCs is found. Using the velocity dispersion measurements, we claim that the difference between the regimes is the nature of the dominant binding force. For the regions in the lower mass range, the dominant force is the external pressure, while in the higher mass range it is the internal gravity of the clouds. We have applied the same method to similar observations ( $\mathrm{GH} \alpha \mathrm{FaS} \& \mathrm{ALMA}$ ) of Arp 236 and NGC 1614 finding similar results.
\end{abstract}

Keywords. Interacting galaxies, star formation, Hiı regions, GMCs.

\section{Introduction}

The process of star formation enhancement in interacting galaxies is postulated but not very well understood. The study of the kinematics of the star forming regions in galaxy mergers turns out to be crucial to clarify if star formation is indeed enhanced, how the extra-nuclear star forming regions are caused, and to characterize the physical parameters of the gas in these regions. Star formation occurs largely inside molecular clouds, and HII regions trace recent star formation. The study of both types of clouds gives us crucial clues to understand the star formation process. The kinematical properties of these clouds help us to clarify what are the drivers of the turbulence, and the connection with the star formation.

Using ASTRODENDRO (Rosolowsky et al. 2008) Zaragoza-Cardiel et al. (2014) were able to measure the radius $(R)$, the velocity dispersion $\left(\sigma_{v}\right)$ and the luminosity for each HII region and GMC observed in the Antennae galaxies. The data used in Zaragoza-Cardiel et al. (2014) is already publicly available. $\dagger$ Based on the luminosity functions, and the virial parameter of HII regions and GMCs in the Antennae galaxies, Zaragoza-Cardiel et al. (2014) conclude that the most massive GMCs are not dissolved after the birth of massive star clusters, but remain as gravitationally bound HıI regions.

$\dagger$ http://vizier.cfa.harvard.edu/viz-bin/VizieR?-source=J/MNRAS/445/1412 

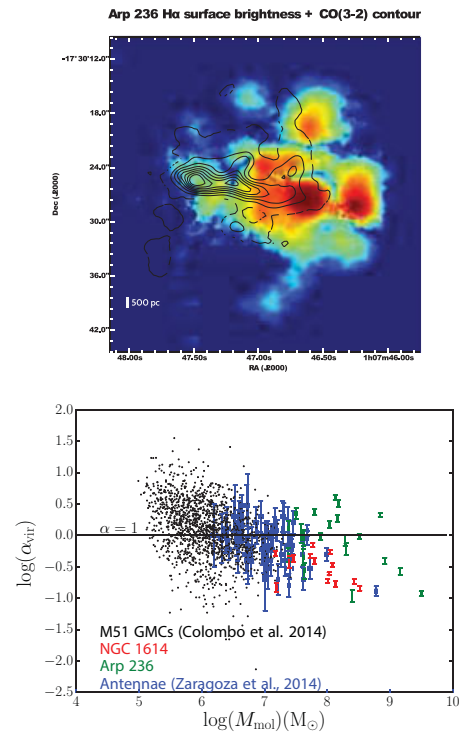
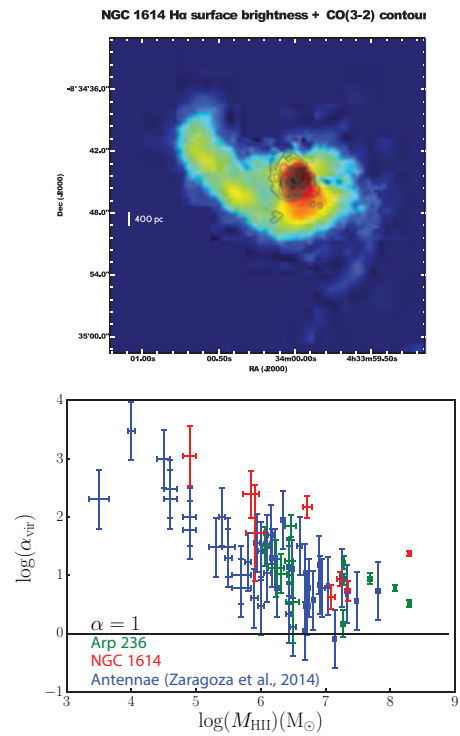

Figure 1. Top left: Arp $236 \mathrm{H} \alpha$ surface brightness $+\mathrm{CO}(3-2)$ contours. Top right: NGC 1614 $\mathrm{H} \alpha$ surface brightness $+\mathrm{CO}(3-2)$ contours. Bottom left: Virial parameter, $\alpha_{\mathrm{vir}}$, versus molecular gas mass, $M_{\mathrm{mol}}$, of GMCs. Bottom right: $\alpha_{\mathrm{vir}}$ versus ionized gas mass, $M_{\mathrm{HII}}$, of HII regions.

\section{HII regions and GMCs in Arp 236 \& NGC 1614}

We have observed Arp 236, and NGC 1614 with GH $\alpha$ FaS and combined these observations with public ALMA data, Saito et al. (2015) for Arp 236, and Sliwa et al. (2014) for NGC 1614. The comparison between $\mathrm{H} \alpha$ and $\mathrm{CO}(3-2)$ observations is shown in Fig. 1 top. Using ASTRODENDRO we have extracted the properties of HiI regions and GMCs in those galaxies.

The virial parameter decreases with the mass of the star forming regions (Fig. 1 bottom), which implies that the more massive the region, the more important is the gravity rather than the external pressure (Bertoldi \& McKee 1992). Thus, the massive population of gravitationally bound HII regions is probably due to the massive population of GMCs.

\section{Conclusions}

We found a population of massive gravitationally bound GMCs and HII regions probably connected based on the luminosity functions and the virial parameter in the Antennae galaxies, Arp 236, and NGC 1614.

\section{References}

Bertoldi, F. \& McKee, C. F. 1992, ApJ, 395, 140

Colombo, D., Hughes, A., Schinnerer, E., et al. 2014, ApJ, 784, 3

Rosolowsky, E. W., Pineda, J. E., Kauffmann, J., \& Goodman, A. A. 2008, ApJ, 679, 1338

Saito, T., Iono, D., Yun, M. S., et al. 2015, ApJ, 803, 60

Sliwa, K., Wilson, C. D., Iono, D., Peck, A., \& Matsushita, S. 2014, ApJL, 796, L15

Zaragoza-Cardiel, J., Font, J., Beckman, J. E., et al. 2014, MNRAS, 445, 1412 